\title{
The effect of diesel knock on a turbocharged diesel engine emissions and top compression ring friction
}

\author{
Anastasios Zavos ${ }^{1 *}$, Pantelis G. Nikolakopoulos ${ }^{1}$, Apostolos Pesyridis ${ }^{2,3}$ and Alasdair \\ Cairns $^{4}$ \\ ${ }^{1}$ Machine Design Laboratory, Department of Mechanical Engineering and Aeronautics, University of \\ Patras, Patras, Greece, 26504 \\ ${ }^{2}$ Metapower Limited, Northwood, Middlesex, HA6 3LG, UK \\ ${ }^{3}$ College of Engineering, Alasala University, King Fahad Bin Abdulaziz Rd., 31483, Dammam, KSA \\ ${ }^{4}$ University of Nottingham, University Park, NG7 2RD, UK
}

\begin{abstract}
Turbocharged engines with direct injection offer a significant contribution to engine downsizing technology. However, there remain many unsolved and ambitious issues concerning knocking and pre-ignition. Therefore, detailed understanding of the top compression ring lubrication and fuel economy is critical. This paper focuses on the tribological performance of the top compression ring under partially lubricated conditions caused by diesel knock in a turbocharged diesel engine. A mixedhydrodynamics model was built including multi-phase flow and asperity interactions with realistic boundary conditions. The study shows that frictional power losses in the compression ring-liner contact increased owing to diesel knock and starved conditions in a turbocharged gasoline engine. This finding indicates that the control of knocking combined with the inlet flow conditions can help to mitigate fuel economy and emissions in ringliner conjunction.
\end{abstract}

\section{Introduction}

One opportunity to reduce fuel consumption and to increase engine peak power of SI engines is downsizing. Turbo charging in conjunction with direct injection are the key aspects for enabling this technology [1]. Therefore, the development of a high-performance powertrain is of prime interest. Nowadays, it is widely known that the piston-cylinder system is a major contributor to the overall parasitic losses [2, 3]. According to Richardson [4], the top compression ring friction is considered to be between $13 \%$ and $40 \%$ of all the parasitic losses depending on the engine and the running conditions. As a result, detailed understanding of ring-pack tribodynamics must be achieved.

A vast number of researchers consider that the major source of power loss within the Internal Combustion (IC) engines is the friction (viscous and boundary) between the piston rings and the cylinder bore $[5,6]$. Typically, it is well known that there are three rings fit into the piston, i.e. the compression ring, the scraper ring and the oil control ring. As the piston reciprocates along the cylinder bore, the top compression ring is pushed radially against the cylinder liner with variable forces including the pretension force and the gas force generated from the in-cylinder pressure. For this reason, the friction losses increased while the surfaceto-surface contact occurs. Therefore, the deep insight into transient nature of ring-liner

* Corresponding author: zavos@upatras.gr 
lubrication should be investigated. The physics of oil flow in the ring-liner interface is a multi-variate problem. A key research in the piston ring lubrication field was that of Jeng [7] who investigated ring tribology under starved conditions. Additionally, the suitable inlet condition is essential for realistic prediction of starvation. For this reason, much attention has been devoted to the investigation of this phenomenon including power loss and lubricant availability [8-10].

In the case of turbocharged SI engines, the key phenomena such as knocking and preignition are predominant [11,12], which affect both frictional and integrity performance of the piston assembly system. The current paper attempts to address the issues of frictional losses particularly in the top compression ring- liner contact. The geometric dimensions of the compression ring-cylinder model were obtained from a turbocharged engine with a focus on engine knocking. Flow simulations were performed solving the 2D Navier-Stokes equations, vapor transport and asperity interactions. The present study combines fully flooded and starved conditions with diesel knock, thus providing a more realistic analysis. The effect of "coating-lubricant"' subsystem on boundary friction was also investigated.

\section{ANALYSIS OF PISTON RING-LINER CONTACT}

In this analysis, a 2D CFD model was built using the Ansys FLUENT with the following configurations:

- Two phase flow was solved based on Navier-Stokes equation and vapour transport approach with no artificial boundary conditions.

- The flow is laminar, and the lubricant viscosity was changed according to the pressure into the contact.

- Under mixed conditions of lubrication, the load of asperities was predicted from the stochastic model of Greenwood-Tripp [13].

For hydrodynamic lubrication, the momentum equation, was coupled with the continuity equation and solved. The Finite Volume Method (FVM) was used through FLUENT code, where the domain was discretized into a finite set of control volumes. The following expressions are represented as follows: [14]:

The equation for continuity equation and the conservation of momentum are given as

$$
\begin{gathered}
\frac{D \rho}{D t}+\rho \nabla \vec{V}=0 \\
\rho \frac{D \vec{V}}{D t}=-\nabla p+\nabla \cdot\left(\overline{\tau_{l \jmath}}\right)+\vec{F}
\end{gathered}
$$

With regard to the cavitation region, the Rayleigh-Plesset equation was coupled with Navier-Stokes equation This puts more realistic lubricated conditions in the diverging ring area. The corresponding parameters for cavitation flow were obtained using the work of Singhal et al. [15]. Further information can be also found in publication of Zavos and Nikolakopoulos [16]. 


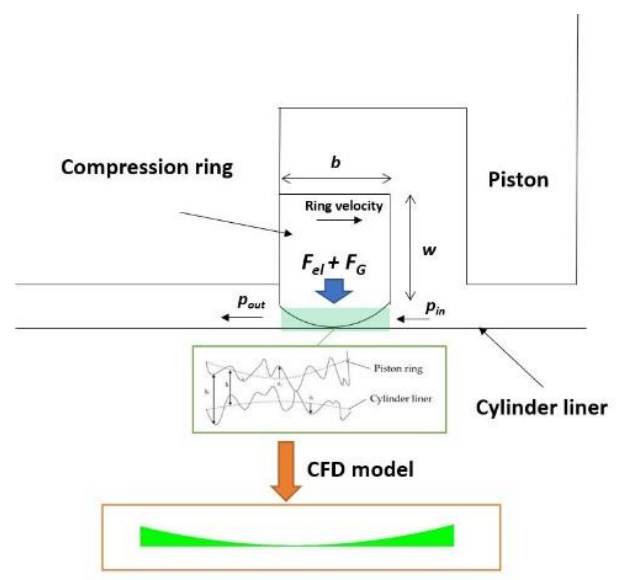

Fig. 1. The mechanism of ring-liner conjunction.

Fig. 1 described the mechanism of piston ring-liner conjunction into the piston groove. The main forces behind the ring are presented and defined here. The back-gas force is calculated as:

$$
F_{G}=\pi d_{c y l} b p_{b k}(\varphi)
$$

The tension force is also expressed as:

$$
F_{e l}=\pi d_{c y l} b p_{e l}
$$

where the elastic pressure is $p_{e l}=\frac{d_{g a p} E_{r} I_{r}}{3 \pi b\left(\frac{d_{c y l}}{2}\right)^{4}}$ and the top ring cross section is $I_{r}=\frac{b w^{3}}{12}$.

The ring profile reciprocates along the cylinder liner related to the piston sliding velocity. This velocity is taken as:

$$
U_{p}(\varphi)=r \omega\left(\sin \varphi+\frac{\lambda_{C R}}{2} \sin 2 \varphi\right)
$$

where $r$ is the crank-pin radius, $\omega$ is the rotational engine speed, $\varphi$ is the crank angle and $\lambda_{C R}$ is the control ratio. The film thickness between the ring and the liner can be approximated as:

$$
h(x, t)=h_{\min }(t)+h_{s}(x)
$$

where $h_{\min }(t)$ is the minimum film and $h_{s}(x)$ is the ideal parabolic ring shape. The cylinder liner has also a circular shape, which is not realistic. However, this case was taken here, because of the present research focuses on lubrication mechanisms into the contact under full and starved conditions when engine knock occurs, which is appropriate for the piston ring tribology involved here.

Under hydrodynamic lubrication during the ring sliding motion, the ring load capacity from the lubricant film is also defined as:

$$
W_{h}=\pi d_{c y l} \int_{-b / 2}^{b / 2} p_{h} d x d y
$$




\subsection{Mixed lubrication}

The stochastic model of Greenwood-Tripp [13] was used to predict the load of asperities and contact area, when the lubricant film is limited $(1 \leq \lambda<3)$. Considering that the asperity height distribution was Gaussian, the generated contact pressure can be calculated as: $p_{c}=$ $\frac{W_{c}}{A_{c}}$, where $W_{c}=\frac{16 \sqrt{2}}{15} \pi(\zeta \kappa \sigma)^{2} \sqrt{\frac{\sigma}{\kappa}} E^{\prime} A F_{5 / 2}(\lambda)$ is the load carried by the asperities and $A_{c}=$ $\pi^{2}(\zeta \kappa \sigma)^{2} A F_{2}(\lambda)$ is the corresponding contact area. The probability distribution of asperity heights $F_{5 / 2}(\lambda), F_{2}(\lambda)$ was evaluated by a fifth-order polynomial curve.

\subsection{Lubricant viscosity}

The lubricant viscosity was evaluated as a relationship of the pressure by the Houpert expression [17]:

$$
\mu=\mu_{o} \exp \left\{\left(\ln \mu_{o}+9.67\right)\left[\left(1+\frac{p_{h y d}-p_{a t m}}{1.98 \times 10^{8}}\right)^{Z_{o}}-1\right]\right\}
$$

where the parameter $Z_{o}$ was defined by Gohar and Rahnejat [18]. This four-stroke turbocharged diesel engine was operated at idle speed of $2000 \mathrm{rpm}$ and lubricant temperature of $80 \mathrm{oC}$. This temperature corresponds to warm engine conditions by New European Driving Cycle (NEDC). The simulations presented here were provided for an isothermal analysis. Fig. 2 shows the variation of lubricant viscosity for different temperatures. The chosen lubricant was Titan XTR 5W30.

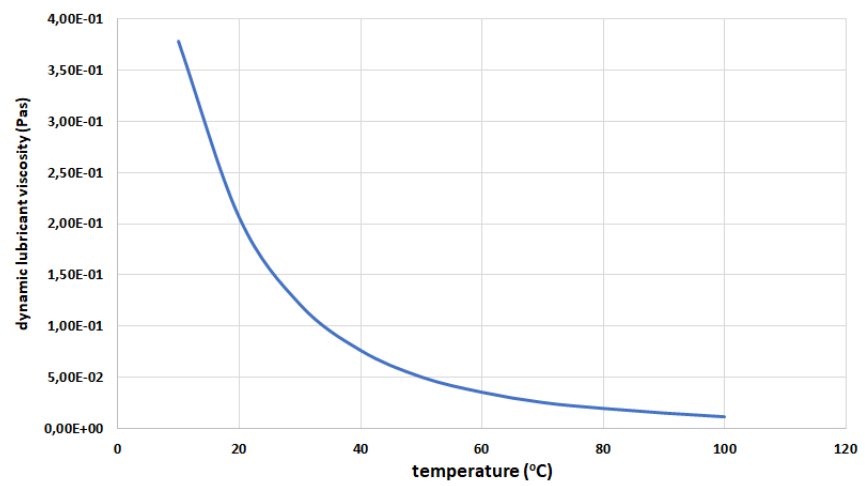

Fig. 2. Dynamic lubricant viscosity for Titan XTR 5 W30 at different lubricant temperatures.

\subsection{Ring friction}

The ring friction can be expressed as:

$$
f_{\text {tot }}=f_{v}+f_{b}
$$

where the viscous friction is given as:

$$
f_{f l}=\left| \pm \frac{h}{2} \nabla p-\Delta \vec{V} \frac{\mu}{h}\right|\left(A-A_{c}\right)
$$


while the boundary friction is:

$$
f_{b}=\tau_{o} A_{c}+\mu_{a s p} W_{c}
$$

in which $\tau_{o}$ is the non-Newtonian Eyring shear stress with value of $2 \mathrm{MPa}$ and $\mu_{\text {asp }}$ is the boundary shear strength of contact asperities. Tomanik et al. [18] reported the tribological performance of several coated piston rings for different lubricants. It was found that measured boundary coefficient of chromium-plated piston ring can be varied between 0.12 and 0.04 . In this analysis, the impact of boundary coefficient on boundary friction was investigated under starved conditions. This trend made in order to model more realistic contact conditions.

\section{CFD model}

Fig. 3 presents the input flow conditions. During the ring motion, the in-cylinder and outlet pressures are used as inputs to the simulation. The outlet gas pressure is assumed to be the atmospheric pressure. Practically, modelling throughout Navier Stokes and Rayleigh-Plesset expression, the swirl flow in the inlet position can be confirmed. In the divergent region as the lubricant passing, cavitation can be also occurred. The boundary conditions are presented here:

$$
\begin{aligned}
& p\left(x, \frac{b}{2}\right)=\left\{\begin{array}{cl}
p_{\text {in }}=p_{c} & \text { for upstoke } \\
p_{\text {out }}=p_{c} & \text { for downstroke }
\end{array}\right. \\
& p\left(x,-\frac{b}{2}\right)=\left\{\begin{array}{cl}
p_{\text {out }}=p_{a} & \text { for upstroke } \\
p_{\text {in }}=p_{a} & \text { for downstroke }
\end{array}\right.
\end{aligned}
$$

The pressure-based mixture model was used, while the velocity-pressure coupling was solved using the Semi-implicit Method for Pressure-linked Equations (SIMPLE) algorithm. A total number of 30,000 quadrilateral volumes were used here. Grid sensitivity tests were obtained to improve accuracy. The time step was also defined as $10^{-3} \mathrm{sec}$.

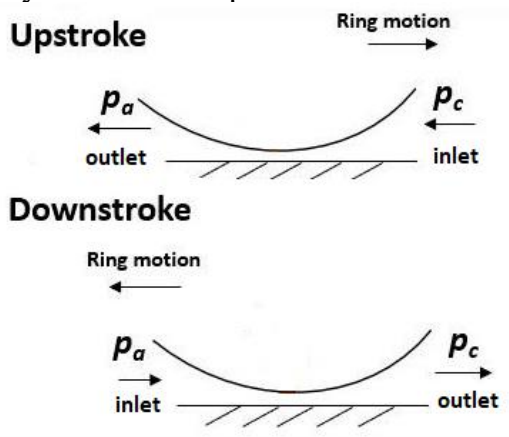

Fig. 3. Boundary conditions in CFD model.

\section{Results and Discussion}

The studied compression ring was from a four-stroke turbocharged diesel engine. Table 1 lists the basic engine data. The ring dimensions, the material properties and the roughness parameters are also presented. The input combustion pressure with engine knock is illustrated 
in Fig. 4. The in-cylinder pressure measurements with diesel knock were obtained directly at engine speed of $2000 \mathrm{rpm}$; thus, we try to model these realistic engine conditions.

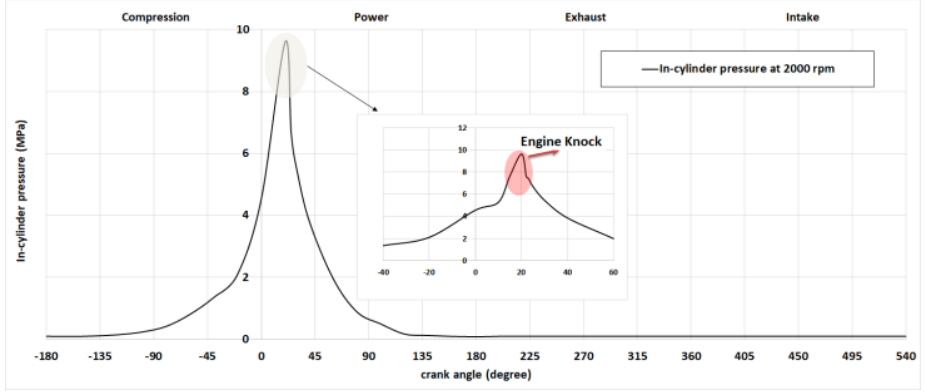

Fig.4. Combustion pressure with engine knock at $2000 \mathrm{rpm}$.

\begin{tabular}{|c|c|c|}
\hline Parameter & Value & Unit \\
\hline Ring material & Steel & - \\
\hline Crank-pin radius & 0,031 & $\mathrm{~m}$ \\
\hline Connecting rod length & 0,10 & $\mathrm{~m}$ \\
\hline Bore nominal radius & 0,048 & $\mathrm{~m}$ \\
\hline Ring face width & 0,0015 & $\mathrm{~m}$ \\
\hline Ring radial width & 0,0035 & $\mathrm{~m}$ \\
\hline Ring roughness & 0,45 & $\mu \mathrm{m}$ \\
\hline
\end{tabular}

Table 1. Engine data.

Fig. 5 shows the variation of minimum film thickness at $2000 \mathrm{rpm}$. Sealing is a significant point of ring operation. Therefore, it is important to investigate the inlet flow of the lubricant through the minimum ring-liner clearance. Fully flooded and starved conditions showed very thin films at the TDC reversal, where the in-cylinder pressures are the highest due to knocking. The mixed regime of lubrication is plotted in the figure using the red bolt line $(\lambda=1)$ and the red dotted line $(\lambda=3)$.

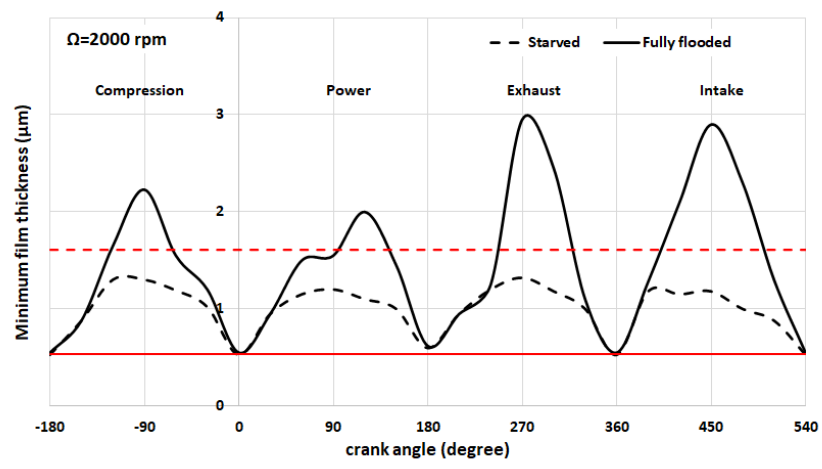

Fig. 5. Effect of inlet flow conditions on the minimum lubricant thickness at $2000 \mathrm{rpm}$. 
However, as the engine speed is increased, thicker film is obtained for fully flooded conditions during the middle of each stroke. On the other hand, starvation has a dominant impact during mid-stroke. This is obvious at the higher piston speeds. Under these run conditions here, it is obvious that partially flooded (starved) inlet was attained. This methodology is more realistic and without artificially input flow conditions (fully flooded). In more details, analysis of flow in the inlet wedge of the conjunction has shown recirculating (swirl) flow in the inset area to the Fig. 6. This trend was reported firstly to the work of Shahmohamadi et al. [8], where the input CFD conditions are compared by Tipei's analytical approach [10].

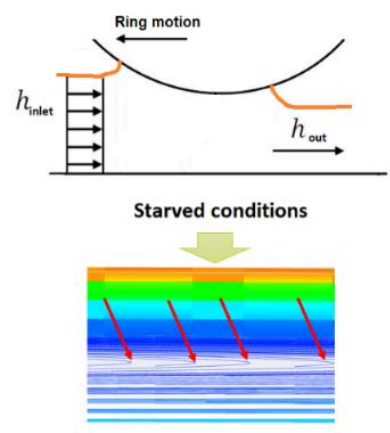

Fig. 6. Analysis of starvation mechanism (swirl flow).

The impact of inlet conditions on boundary friction is presented in Fig. 7. The increment was significant $8.9 \%$ per cent at the piston reversals for starved conditions. It can be seen that the starvation corresponds to higher power loss due to thin lubricant films when engine knock occurs. This is obvious in Fig. 5. As the piston speed is increased, it is clear that by taking into account the effect of starvation, there is a greater contribution during mid-stroke. This trend can lead to increased total friction. Therefore, friction losses are predominant. This means that the knocking-starvation combination may affect negatively the engine efficiency in term of power loss.

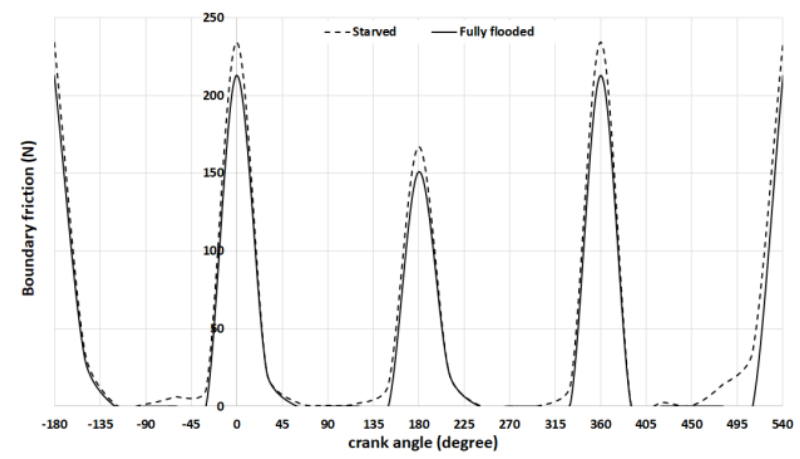

Fig. 7. Effect of inlet flow conditions on boundary friction at $2000 \mathrm{rpm}$.

The effect of the coated ring profile on generated boundary friction is shown in Fig. 8. Clearly, coated compression rings lead to lower friction values due to combined surface lubricant additives interaction and the associated mechanisms of the tribofilm formation. In more details, PVD coated ring has the lower asperity friction of $38,04 \%$ than uncoated steel ring. This trend is obvious owing to the lower boundary coefficient of 0,08 when PVD coating react with a synthetic lubricant. Therefore, compression ring coating has dominant role on contact friction and wear under different running conditions. Ideally, optimisation of 
the compression ring surface properties is needed to address towards for the overall improvement of the engine performance.

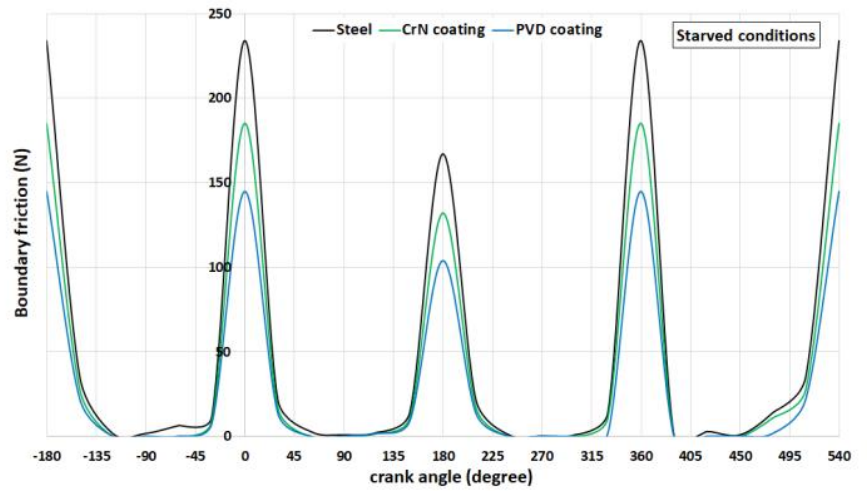

Fig. 8. Effect of coatings on boundary friction at $2000 \mathrm{rpm}$.

\section{Conclusions}

The model presents the tribological performance in the top compression ring under fully and starved lubrication conditions including diesel knock. The mainly contributions from this study are summarized as follows:

- Starvation shows lower films. The most significant effect on generated boundary friction occurs at the piston reversals. The mechanism of reverse and swirl flow in the inlet zone is the source of starvation.

- The current model predicted lower boundary friction using different type of coatings under same starved conditions. Boundary friction coefficient depends on the combined surface - lubricant additives interaction and the associated mechanisms of the tribofilm formation. This investigation needs further action under experimental and numerical methods.

\section{References}

1. R. Martinez-Botas, A. Pesiridis. Y. MingYang, Science China Technological Sciences 54, 318 (2011).

2. K. Holmberg, P. Andersson, A. Erdemir, Tribology International 47, 221 (2012).

3. A. Zavos, P.G. Nikolakopoulos, Tribology International 128, 214 (2018).

4. D. E. Richardson, Transactions-American Society of Mechanical Engineers Journal of Engineering for Gas Turbines and Power 122, 506 (2000).

5. R. I. Taylor, N. Morgan, R. Mainwaring, T. Davenport, Proc. of the Institution of Mechanical Engineers, Part J: Journal of Engineering Tribology 234, 1563 (2020).

6. A. Zavos, P. G. Nikolakopoulos, Tribology-Materials, Surfaces \& Interfaces, (2021).

7. Y. R. Jeng, Tribology Transactions 35, 707 (1992).

8. H. Shahmohamadi, M. Mohammadpour, R. Rahmani, H. Rahnejat, C. P. Garner, S. Howell-Smith, Tribology International 90, 164 (2015). 
9. S. R. Bewsher, M. Mohammadpour, H. Rahnejat, G. Offner, O. Knaus, Proceedings of the Institution of Mechanical Engineers, Part J: Journal of Engineering Tribology 233, 112 (2019).

10. N. Tipei, Trans. ASME. J. Lubr. Technol. 90, 8 (1968).

11. H. Vafamehr, A. Cairns, O. Sampson, M.M. Koupaie, Applied Energy 179, 687 (2016).

12. Y. Liu, A. Kuznetsov, B. Sa, Applied Sciences 11, 7603 (2021).

13. J.A. Greenwood, J.H. Tripp, Proc Inst Mech Eng. 185, 625 (1970).

14. F. White, 2nd ed. New York (NY), McGraw-Hill (1991).

15. A. K. Singhal, M. M Athavale, H. Li, Y. Jiang, J. Fluids Eng. 124, 617 (2002).

16. A. Zavos, P. G. Nikolakopoulos, Lubrication Science 28, 475 (2016).

17. R. Gohar, H. Rahnejat, World Scientific (2018).

18. E. Tomanik, F. Profito, B. Sheets, R. Souza, Tribology International 149, 105514 (2018). 